\title{
Self-Similar Flow under the Action of Monochromatic Radiation Behind a Cylindrical MHD Shock in a Non-Ideal Gas
}

\author{
J. P. Vishwakarma*, Vijay Kumar Pandey \\ Dept. of Mathematics \& Statistics, D.D.U. Gorakhpur University Gorakhpur, 273009, India
}

\begin{abstract}
Similarity solutions are obtained for one-dimensional flow under the action of monochromatic radiation behind a cylindrical magnetogasdynamic shock wave propagating in a non-ideal gas in presence of an axial magnetic field. The initial density of the medium and initial magnetic field are assumed to be constant. It is investigated that the presence of the magnetic field or the non-idealness of the gas decays the shock wave, and when the initial magnetic field is strong the non-idealness of the gas affects the velocity and pressure profiles significantly. Also, it is observed that the flow-variables behind the shock are affected significantly, by an increase in the parameter of radiation, when the initial magnetic field is strong. It is, therefore, inferred that the effect of the non-idealness of the gas and of the monochromatic radiation on the shock propagation become more significant when the strength of the initial magnetic field is increased.
\end{abstract}

Keywords Shock Wave, Self-similar Flow, Non-ideal Gas, Magnetohydrodynamics, Monochromatic Radiation

\section{Introduction}

In gasdynamics, if the effects of radiation are taken into account, the basic non-linear equations are of a very complicated form and therefore it is essential to establish approximations which are physically accurate and can afford considerable simplifications. The problem of the interaction of radiation with gasdynamics has been studied by many authors by using the method of self-similarity developed by Sedov[1]. Marshak[2] studied the effect of radiation on the shock propagation by introducing the radiation diffusion approximation. He solved both the cases of constant density and constant pressure fields without invoking conditions of self-similarity. Using the same mode of radiation, Elliott[3] discussed the conditions leading to self-similarity with a specified functional form of the mean free-path of radiation and obtained a solution for self-similar explosions. Wang[4], Helliwell[5] and Nicastro[6] treated the problems of radiating walls, either stationary or moving, generating shock at the head of self-similar flow-fields. Assuming the shock to be isothermal and transparent the self-similar solution of the central explosions in stars has been obtained by Ray and Bhowmick[7] including the effects of radiation.

The self-similar solutions have been used by Khudyakov

* Corresponding author:

jpv_univgkp@yahoo.com (J. P. Vishwakarma)

Published online at http://journal.sapub.org/am

Copyright ( 2012 Scientific \& Academic Publishing. All Rights Reserved
[8] to discuss the problem of the motion of a gas under the action of monochromatic radiation. Khudyakov[8] has considered that a homogeneous gas at rest occupies a half-space bounded by a fixed plane wall and assumed that a radiation flux moves through the gas in the direction of the wall with a constant intensity $j_{0}$ per unit area. From the instant of arrival of the radiation at the wall a shock wave is assumed to propagate out from the wall in the direction opposite to the radiation flux. The radiation flux is absorbed in the zone between the shock wave and the wall, and it is not absorbed in the undisturbed medium. It is also assumed that the gas itself does not radiate. Zheltukhin[9] has developed a family of exact solutions of one dimensional motion (plane, cylindrical or spherical symmetry) of a gas taking into account of the absorption of monochromatic radiation. Nath and Takhar[10] and Nath[11] have studied the propagation of cylindrical shock waves in a gas under the action of monochromatic radiation when the medium is rotating or non-rotating.

Since at high temperatures that prevail in the problems associated with shock wave a gas is ionized, electromagnetic effects may also be significant. A complete analysis of such a problem should therefore consist of the study of the gasdynamic flow and the electromagnetic filed simultaneously. The study of the propagation of cylindrical shock waves in a conducting gas in presence of an axial or azimuthal magnetic field is relevant to the experiments on pinch effect, exploding wires, and so forth. This problem both in the uniform or non-uniform ideal gas was undertaken by many investigators, for example, Pai[12], Cole and 
Grefinger[13], Sakurai[14], Bhutani[15], Christer and Helliwell[16], Deb Ray[17] and Vishwakarma and Yadav[18]. Nath[19] and Shinde[20] have considered the effect of magnetic field on the propagation of cylindrical shock waves under the action of monochromatic radiation by using the method of self-similarity. In all of these works, the medium is assumed to be a gas obeying the equation of state of an ideal gas.

Because of high pressure and density that generally occur behind a shock wave, produced by an explosion, the assumption that the gas is ideal is no more valid. The popular alternative to the ideal gas is a simplified van der Waals model. Roberts and $\mathrm{Wu}[21]$ and $\mathrm{Wu}$ and Roberts[22] adopted this model to discuss the shock wave theory of sonoluminescence. In the present work, we too adopt this as our model of a non-ideal gas to discover how deviations from the ideal gas can affect the self-similar solutions for the flow with monochromatic radiation behind a magnetogasdynamic cylindrical shock wave propagating in a non-ideal gas permeated by an axial magnetic field. The non-ideal gas is assumed to have infinite electrical conductivity and constant specific heats. The initial density of the medium and the initial magnetic field are assumed to be constant.

Effects of a change in the strength of initial magnetic field, in the parameter of non-idealness of the gas and in the parameter of the flux of monochromatic radiation on the shock propagation are investigated. It is observed that the effects of non-idealness of the gas and the monochromatic radiation on the flow variables in the flow-field behind the shock are significant when the initial magnetic field is strong. The present work may be considered as an extension of the work of Nath[11] by taking the medium a non-ideal gas in place of an ideal gas.

\section{Basic Equations and Boundary Conditions}

The fundamental equations for cylindrically symmetric motion of a non-ideal gas under the action of monochromatic radiation and axial magnetic field, neglecting heat-conduction, viscosity and radiation of the medium, may be written as (Khudyakov[8], Greifinger and Cole[13], Nath[19], Zedan[23])

$$
\begin{gathered}
\frac{\partial \rho}{\partial \mathrm{t}}+\mathrm{v} \frac{\partial \rho}{\partial \mathrm{r}}+\rho \frac{\partial \mathrm{v}}{\partial \mathrm{r}}+\frac{\rho \mathrm{v}}{\mathrm{r}}=0 \\
\rho \frac{\partial \mathrm{v}}{\partial \mathrm{t}}+\rho \mathrm{v} \frac{\partial \mathrm{v}}{\partial \mathrm{r}}+\frac{\partial \mathrm{p}}{\partial \mathrm{r}}+\mathrm{h} \frac{\partial \mathrm{h}}{\partial \mathrm{r}}=\mathrm{O}, \\
\frac{\partial \mathrm{h}}{\partial \mathrm{t}}+\mathrm{v} \frac{\partial \mathrm{h}}{\partial \mathrm{r}}+\mathrm{h} \frac{\partial \mathrm{v}}{\partial \mathrm{r}}+\frac{\mathrm{hv}}{\mathrm{r}}=0 \\
\frac{\partial \mathrm{e}}{\partial \mathrm{t}}+\mathrm{v} \frac{\partial \mathrm{e}}{\partial \mathrm{r}}-\frac{\mathrm{p}}{\rho^{2}}\left(\frac{\partial \rho}{\partial \mathrm{t}}+\mathrm{v} \frac{\partial \rho}{\partial \mathrm{r}}\right)=\frac{1}{\rho r} \frac{\partial}{\partial \mathrm{r}}(\mathrm{jr}), \\
\frac{\partial \mathrm{j}}{\partial \mathrm{r}}=\mathrm{Kj},
\end{gathered}
$$

where $\rho, v, p, h, e$ and $j$ are the density, radial velocity, pressure, axial magnetic field, internal energy per unit mass and the monochromatic radiation at distance $r$ from the axis at time $\mathrm{t}$, and $\mathrm{K}$ is the absorption coefficient. Here the magnetic permeability is taken to be unity and the electrical conductivity to be infinite.

Most of the phenomena associated with shock wave arise in extreme conditions under which the ideal gas is not a sufficiently accurate description. To discover how deviations from the ideal gas can affect the flow behind a shock wave, we adopt a simple model. We assume that the gas obeys a simplified van der waals equation of state of the form (Roberts and $\mathrm{Wu}[21], \mathrm{Wu}$ and Roberts[22])

$$
\mathrm{p}=\frac{\overline{\mathrm{R}} \rho \mathrm{T}}{1-\mathrm{b} \rho}, \quad \mathrm{e}=\mathrm{C}_{\mathrm{v}} \mathrm{T}=\frac{\mathrm{p}(1-\mathrm{b} \rho)}{\rho(\gamma-1)},
$$

where $\bar{R}$ is the gas constant and $C_{v}=\bar{R} /(\gamma-1)$ is the specific heat at constant volume and $\gamma$ is the ratio of specific heats. The constant $b$ is the "van der Waals excluded volume", it places a limit, $\rho_{\max }=1 / \mathrm{b}$, on the density of the gas.

The absorption coefficient $\mathrm{K}$ is considered to vary as (Khudyakov[8], Nath[19], Nath and Takhar[10])

$$
\mathrm{K}=\mathrm{K}_{0} \rho^{\mathrm{n}} \mathrm{p}^{\mathrm{m}} \mathrm{j}^{\mathrm{q}} \mathrm{r}^{\mathrm{s}} \mathrm{t}^{1}
$$

where the coefficient $\mathrm{K}_{\mathrm{o}}$ is a dimensional constant and the exponents $\mathrm{n}, \mathrm{m}, \mathrm{q}, \mathrm{s}, \mathrm{l}$ are rational numbers.

A diverging cylindrical shock is assumed to be propagating in the perfectly conducting non-ideal gas with constant density, in presence of a uniform axial magnetic field. The jump conditions across the shock front which is transparent for the radiation flux, are as

$$
\begin{gathered}
\rho_{1}\left(D-v_{1}\right)=\rho_{0} D, \\
h_{1}\left(D-v_{1}\right)=h_{0} D, \\
p_{1}+\rho_{1}\left(D-v_{1}\right)^{2}+\frac{1}{2} h_{1}^{2}=p_{0}+\rho_{0} D^{2}+\frac{1}{2} h_{0}^{2}, \\
e_{1}+\frac{p_{1}}{\rho_{1}}+\frac{1}{2}\left(D-v_{1}\right)^{2}+\frac{h_{1}^{2}}{\rho_{1}}=e_{0}+\frac{p_{0}}{\rho_{0}}+\frac{1}{2} D^{2}+\frac{h_{0}^{2}}{\rho_{0}}, \\
j_{1}=j_{0},
\end{gathered}
$$

where the suffices ' 1 ' and ' 0 ' refer to conditions just behind and just ahead of the shock respectively and $D$ is the shock velocity.

If the shock is strong, the boundary conditions (2.8)-(2.12) take the form

$$
\begin{gathered}
\mathrm{v}_{1}=(1-\beta) D, \\
\rho_{1}=\frac{\rho_{0}}{\beta}, \\
\mathrm{p}_{1}=(1-\beta)+\frac{1}{2} \mathrm{M}_{\mathrm{A}}^{-2}\left(1-\frac{1}{\beta^{2}}\right), \\
\mathrm{h}_{1}=\frac{\mathrm{h}_{0}}{\beta}, \\
\mathrm{j}_{1}=\mathrm{j}_{0},
\end{gathered}
$$

where the quantity $\beta \quad(0<\beta<1)$ is obtained by the relation 


$$
\begin{aligned}
& \beta^{3}(\gamma+1)-\left\{\mathrm{M}_{\mathrm{A}}^{-2}(\gamma+2)+2 \overline{\mathrm{b}}+\gamma+1\right\} \beta^{2} \\
& +(\gamma-1) \mathrm{M}_{\mathrm{A}}^{-2} \beta+\overline{\mathrm{b}} \mathrm{M}_{\mathrm{A}}^{-2}=0,
\end{aligned}
$$

$M_{A}=\left(\frac{\rho_{0} D^{2}}{h_{0}^{2}}\right)^{1 / 2}$ being the Alfven-Mach number and $\overline{\mathrm{b}}=\mathrm{b} \rho_{0}$ the parameter of non-idealness.

The dimensions of the constant coefficient $\mathrm{K}_{0}$ in equation (2.7) are given by

$$
\left[\mathrm{K}_{0}\right]=\mathrm{M}^{-\mathrm{n}-\mathrm{m}-\mathrm{q}} \mathrm{L}^{3 \mathrm{n}+\mathrm{m}-\mathrm{s}-1} \mathrm{~T}^{2 \mathrm{~m}+3 \mathrm{q}-1}
$$

Following the approach of Sedov[1], we get the conditions under which the formulated problem will have self-similar solutions. The dimensional constants in the present problem will be $\mathrm{h}_{0}\left(\operatorname{or~}_{0}^{1 / 2}\right), \rho_{0}, \mathrm{j}_{0}$, and $\mathrm{K}_{0}$ in which $\mathrm{h}_{0}, \rho_{0}$ and $\mathrm{j}_{0}$ are dependent given by

$$
\mathrm{j}_{0}=\left[\mathrm{h}_{0}\right]^{3}\left[\rho_{0}\right]^{-1 / 2} \text {. }
$$

For self-similarity the radiation absorption coefficient $\mathrm{K}_{0}$ must be dependent on the dimensions of $\mathrm{j}_{0}, \rho_{0}$ which is equivalent to $\mathrm{s}+1=-1$. The self-similar independent dimensionless variable $\lambda$ is taken in the form $\lambda=r / r_{1}$, where

$$
r_{1}=\bar{\beta} j_{0}^{1 / 3} \rho_{0}^{1 / 3} t,
$$

$r_{1}$ being the radius of the shock surface. The value of the constant $\bar{\beta}$ is so chosen that $\lambda=1$ at the shock surface.

\section{Similarity Solutions}

We introduce the following similarity transformations to reduce the equation of motion into ordinary differential equations;

$$
\begin{aligned}
& v=D V(\lambda), \rho=\rho_{0} R(\lambda), h=\rho_{0}^{1 / 2} \operatorname{DH}(\lambda), \\
& p=\rho_{0} D^{2} P(\lambda), j=j_{0} J(\lambda),
\end{aligned}
$$

where $\mathrm{V}, \mathrm{R}, \mathrm{H}, \mathrm{P}$ and $\mathrm{J}$ are functions of the non-dimensional variable (similarity variable) $\lambda=\frac{\mathrm{r}}{\mathrm{r}_{1}}$.

Using the transformations (3.1)-(3.5) the equations of motion take the form

$$
\begin{aligned}
& \mathrm{R}^{\prime} \lambda(\mathrm{V}-\lambda)+\mathrm{R}\left(\mathrm{V}^{\prime} \lambda+\mathrm{V}\right)=0, \\
& \mathrm{P}^{\prime}+\mathrm{H} \mathrm{H}^{\prime}-\mathrm{RV} \mathrm{V}^{\prime}(\lambda-\mathrm{V})=0, \\
& \mathrm{H}^{\prime} \lambda(\mathrm{V}-\lambda)+\mathrm{H}\left(\mathrm{V}^{\prime} \lambda+\mathrm{V}\right)=0, \\
& \mathrm{P}^{\prime}(\mathrm{V}-\lambda)(1-\overline{\mathrm{b}} \mathrm{R})+\frac{\gamma \mathrm{P}}{\lambda}\left(\lambda \mathrm{V}^{\prime}+\mathrm{V}\right) \\
& =\frac{\gamma-1}{\lambda}\left[\lambda \mathrm{J}^{\prime}+\mathrm{J}\right], \\
& \quad \mathrm{J}^{\prime}=\alpha \lambda^{\mathrm{s}} \mathrm{R}^{\mathrm{n}} \mathrm{P}^{\mathrm{m}} \mathrm{J}^{\mathrm{q}+1}, \\
& \text { where } \quad \alpha=\mathrm{K}_{0} \mathrm{j}_{0}^{\mathrm{q}+1} \rho_{0}^{\mathrm{n}+\mathrm{m}-1} \mathrm{D}^{2(\mathrm{~m}-1)+\mathrm{s}}
\end{aligned}
$$

is a dimensionless quantity. The quantity $\alpha$ is taken as the parameter which characterizes the interaction between the gas and the incident radiation flux (Khudyakov[8], Nath[19], Nath and Takhar[10]).

Solving equations (3.6)-(3.10) for $\frac{\mathrm{dV}}{\mathrm{d} \lambda}, \frac{\mathrm{dR}}{\mathrm{d} \lambda}, \frac{\mathrm{dH}}{\mathrm{d} \lambda}$, $\frac{\mathrm{dP}}{\mathrm{d} \lambda}$ and $\frac{\mathrm{d} J}{\mathrm{~d} \lambda}$, we have

$$
\begin{gathered}
\mathrm{V}^{\prime}=\frac{\left[\gamma \mathrm{PV}-(\gamma-1)\left(\alpha \lambda^{\mathrm{s}+1} \mathrm{R}^{\mathrm{n}} \mathrm{P}^{\mathrm{m}} \mathrm{J}^{\mathrm{q}+1}+\mathrm{J}\right)+\mathrm{H}^{2} \mathrm{~V}(1-\overline{\mathrm{b}} \mathrm{R})\right]}{\lambda\left[(\lambda-\mathrm{V})^{2}(1-\overline{\mathrm{b}} \mathrm{R}) \mathrm{R}-\left\{\gamma \mathrm{P}+\mathrm{H}^{2}(1-\overline{\mathrm{b}} \mathrm{R})\right\}\right]} \\
\mathrm{R}^{\prime}=\frac{\mathrm{R}\left(\mathrm{V}^{\prime} \lambda+\mathrm{V}\right)}{\lambda(\lambda-\mathrm{V})} \\
\mathrm{H}^{\prime}=\frac{\mathrm{H}\left(\mathrm{V}^{\prime} \lambda+\mathrm{V}\right)}{\lambda(\lambda-\mathrm{V})} \\
\mathrm{P}^{\prime}=\frac{\left[\gamma \mathrm{P}\left(\lambda \mathrm{V}^{\prime}+\mathrm{V}\right)-(\gamma-1)\left(\lambda \mathrm{J}^{\prime}+\mathrm{J}\right)\right]}{(\lambda-\mathrm{V})(1-\overline{\mathrm{b}} \mathrm{R}) \lambda} \\
\mathrm{J}^{\prime}=\alpha \lambda^{\mathrm{s}} \mathrm{R}^{\mathrm{n}} \mathrm{P}^{\mathrm{m}} \mathrm{J}^{\mathrm{q}+1}
\end{gathered}
$$

The shock conditions (2.11)-(2.15) are transformed into

$$
\begin{gathered}
\mathrm{V}(1)=1-\beta, \\
\mathrm{R}(1)=\frac{1}{\beta}, \\
\mathrm{H}(1)=\frac{1}{\beta} \mathrm{M}_{\mathrm{A}}^{-1}, \\
\mathrm{P}(1)=(1-\beta)+\frac{1}{2} \mathrm{M}_{\mathrm{A}}^{-1}\left(1-\frac{1}{\beta^{2}}\right), \\
\mathrm{J}(1)=1 .
\end{gathered}
$$

At the inner boundary (surface) of the flow-filed behind the shock, the condition is that the velocity of the surface is equal to the normal velocity of the fluid on the surface. This kinematic condition from equation (3.1) can be written as

$$
\mathrm{V}\left(\lambda_{\mathrm{p}}\right)=\lambda_{\mathrm{p}}
$$

where $\lambda_{p}$ is the value of $\lambda$ at the inner surface.

For exhibiting the numerical solutions, it is convenient to write the flow variables in the non-dimensional form as

$$
\begin{aligned}
& \frac{\mathrm{v}}{\mathrm{V}_{1}}=\frac{\mathrm{V}(\lambda)}{\mathrm{V}(1)}, \frac{\rho}{\rho_{1}}=\frac{\mathrm{R}(\lambda)}{\mathrm{R}(1)}, \frac{\mathrm{h}}{\mathrm{h}_{1}}=\frac{\mathrm{H}(\lambda)}{\mathrm{H}(1)}, \\
& \frac{\mathrm{p}}{\mathrm{p}_{1}}=\frac{\mathrm{P}(\lambda)}{\mathrm{P}(1)}, \frac{\mathrm{j}}{\mathrm{j}_{1}}=\frac{\mathrm{J}(\lambda)}{\mathrm{J}(1)} .
\end{aligned}
$$

\section{Results and Discussion}

The set of differential equations (3.12)-(3.16) are numerically integrated with the boundary conditions (3.17)-(3.21) to obtain the non-dimensional variables of the flow-field V, R, H, P and J against the similarity variable $\lambda$ by using the Runge-Kutta method of order four, for the values (Khudyakov[8], Nath[19], Nath and Takhar[10], Ranga Rao and Purohit[24], Singh et. al[25], Vishwakarma and Singh[26]) $\alpha=0.1,0.2 ; \mathrm{n}=-1 / 2 ; \mathrm{m}=3 / 2$; $\mathrm{q}=0 ; \mathrm{s}=1 ; \gamma=5 / 3 ; \mathrm{M}_{\mathrm{A}}^{-2}=0.05,0.06 ; \overline{\mathrm{b}}=0$, 
$0.05,0.1$. The case $\bar{b}=0$ corresponds to the perfect gas case (Nath[19]).

In figure 1 we have plotted the radial velocity $\mathrm{v} / \mathrm{v}_{1}$, in figure 2 the density $\rho / \rho_{1}$, in figure 3 the axial magnetic field $\mathrm{h} / \mathrm{h}_{1}$, in figure 4 the pressure $\mathrm{p} / \mathrm{p}_{1}$ and in figure 5 the radiation flux $\mathrm{j} / \mathrm{j}_{1}$ against the radial distance $r / r_{1}$ in flow-field between the inner expanding surface and the shock surface. Table 1 and table 2 show, respectively, the density ratio across the shock and the position of inner expanding surface for various values of the parameters $\overline{\mathrm{b}}, \mathrm{M}_{\mathrm{A}}^{-2}, \alpha$. This solution shows that all the flow variables the velocity, the density, the pressure, the magnetic field and the radiation flux decrease from highest at the shock front to lowest at the inner expanding surface.

From table 1 and table 2 and figure 1, figure 2, figure 3, figure 4 and figure 5 it is observed that the effects of an increase in the value of $\mathrm{M}_{\mathrm{A}}^{-2}$ (i.e. the effects of an increase in the strength of ambient magnetic field) are

(i) to decrease $x_{p}$, i.e. to increase the distance of inner expanding surface from the shock front. Physically, it means that the gas behind the shock is less compressed, i.e. the shock strength is reduced;

(ii) to decrease the value of $1 / \beta$, i.e. to decrease the shock strength, which is the same as given in (i) above;

(iii) to decrease the value of $\mathrm{v} / \mathrm{v}_{1}$ and $\mathrm{p} / \mathrm{p}_{1}$; and to increase the value of $\mathrm{j} / \mathrm{j}_{1}$ at any point in the flow-field behind the shock; and

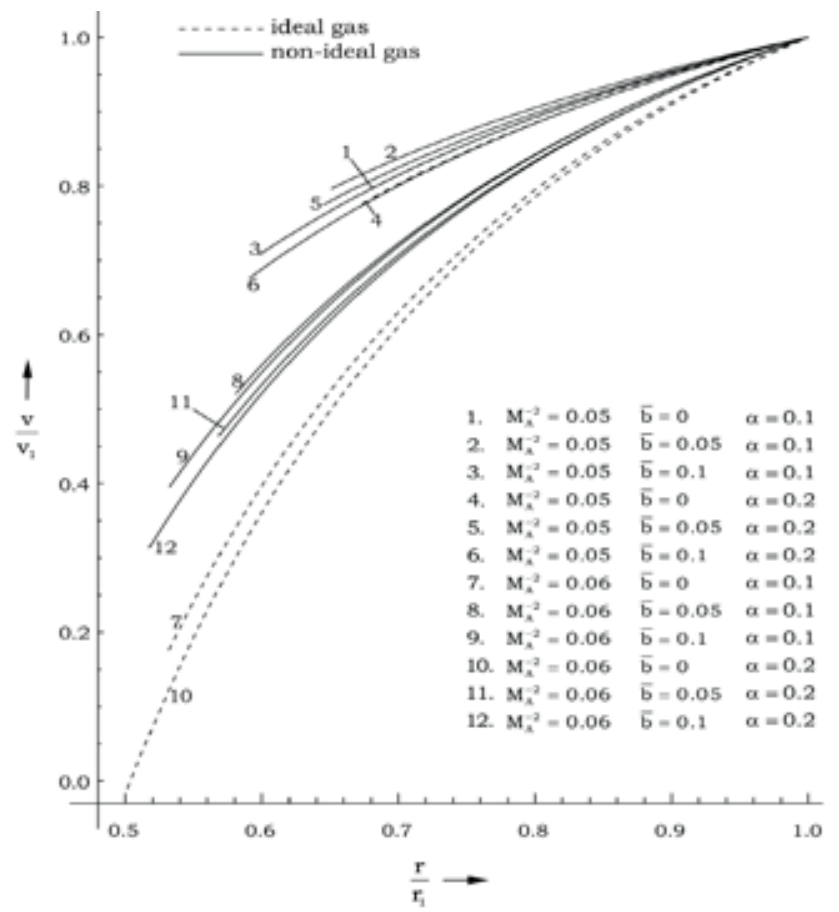

Figure 1. Variation of radial fluid velocity $v / v_{1}$ with radial distance $r / r_{1}$ in the flow-field behind the shock for different values of Alfven-Mach number $M_{A}^{-2}$, parameter of non-idealness of the gas $\bar{b}$ and parameter of radiation $\alpha$ (iv) to enhance the effect of non-idealness of the gas on the profiles of $\mathrm{p} / \mathrm{p}_{1}$.

Thus the presence of magnetic field decays the shock wave and enhances the effect of non-idealness of the gas on the pressure profiles behind the shock.

The effects of an increase in the value of the parameter of the non-idealness of the gas $\bar{b}$ are

(i) to increase the distance of the inner expanding surface from the shock front when $M_{A}^{-2}=0.05$ (see table 2);

(ii) to decrease the value of $1 / \beta$ (table 1 ), i.e. to decrease the shock strength. Therefore the non-idealness of the gas has decaying effect on the shock wave;

(iii) to decrease the value of $\mathrm{j} / \mathrm{j}_{1}$ at any point in the flow-field behind the shock (see figure 5); and

(iv) to change the values of $\mathrm{v} / \mathrm{v}_{1}$ and $\mathrm{p} / \mathrm{p}_{1}$ significantly at any point in the flow-field behind the shock when the magnetic field is strong $\left(\mathrm{M}_{\mathrm{A}}^{-2}=0.06\right)$.

Thus the non-idealness of the gas decays the shock wave, and affects the velocity and pressure profiles significantly when the initial magnetic field is strong.

Effects of an increase in the radiation parameter $\alpha$ are

(i) to decrease $x_{p}$ (table 2), i.e. to decrease the shock strength; and

(ii) to decrease $\mathrm{v} / \mathrm{v}_{1}, \mathrm{p} / \mathrm{p}_{1}, \mathrm{j} / \mathrm{j}_{1}$ at any point in the flow-field behind the shock. This decrease in these flow-variables are enhanced when the initial magnetic field is strong $\left(\mathrm{M}_{\mathrm{A}}^{-2}=0.06\right)$.

The above effects show that the monochromatic radiation is more absorbed by the gas in the flow-field behind the shock by an increase in the parameter $\alpha$.

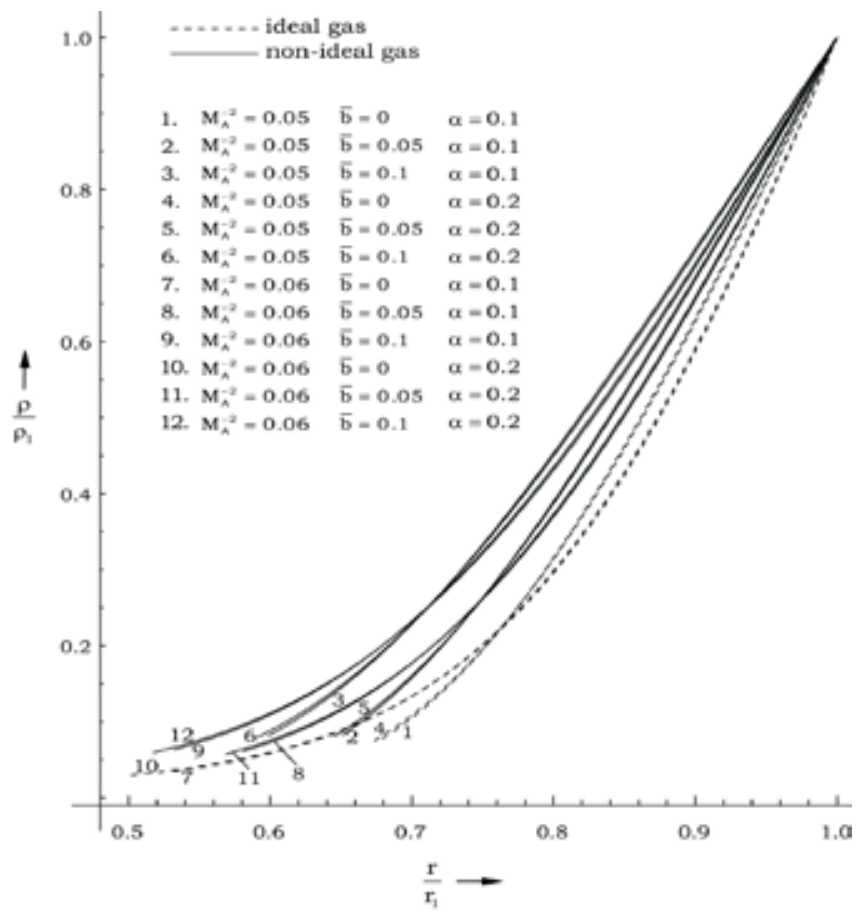

Figure 2. Variation of density $\rho / \rho_{1}$ with radial distance $r / r_{1}$ in the flow-field behind the shock for different values of Alfven-Mach number $M_{A}^{-2}$, parameter of non-idealness of the gas $\bar{b}$ and parameter of radiation $\alpha$ 


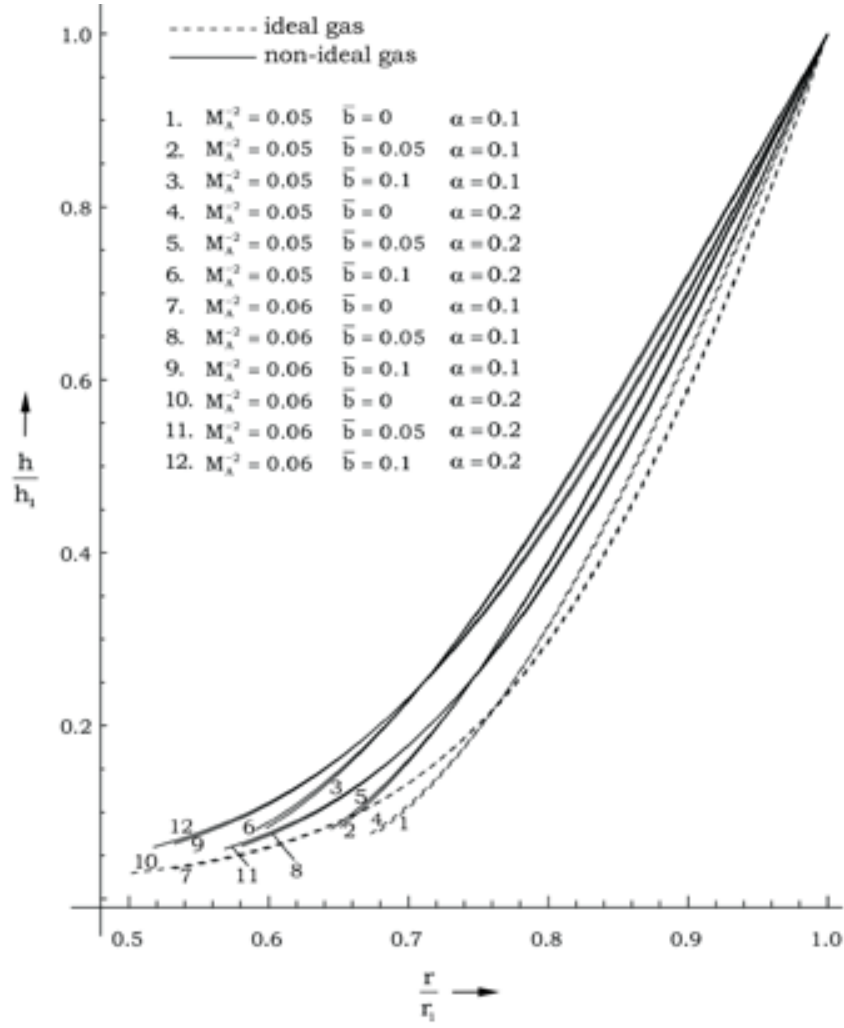

Figure 3. Variation of axial magnetic field $\mathrm{h} / \mathrm{h}_{1}$ with radial distance $\mathrm{r} / \mathrm{r}_{1}$ in the flow-field behind the shock for different values of Alfven-Mach number $M_{A}^{-2}$, parameter of non-idealness of the gas $\bar{b}$ and parameter of radiation

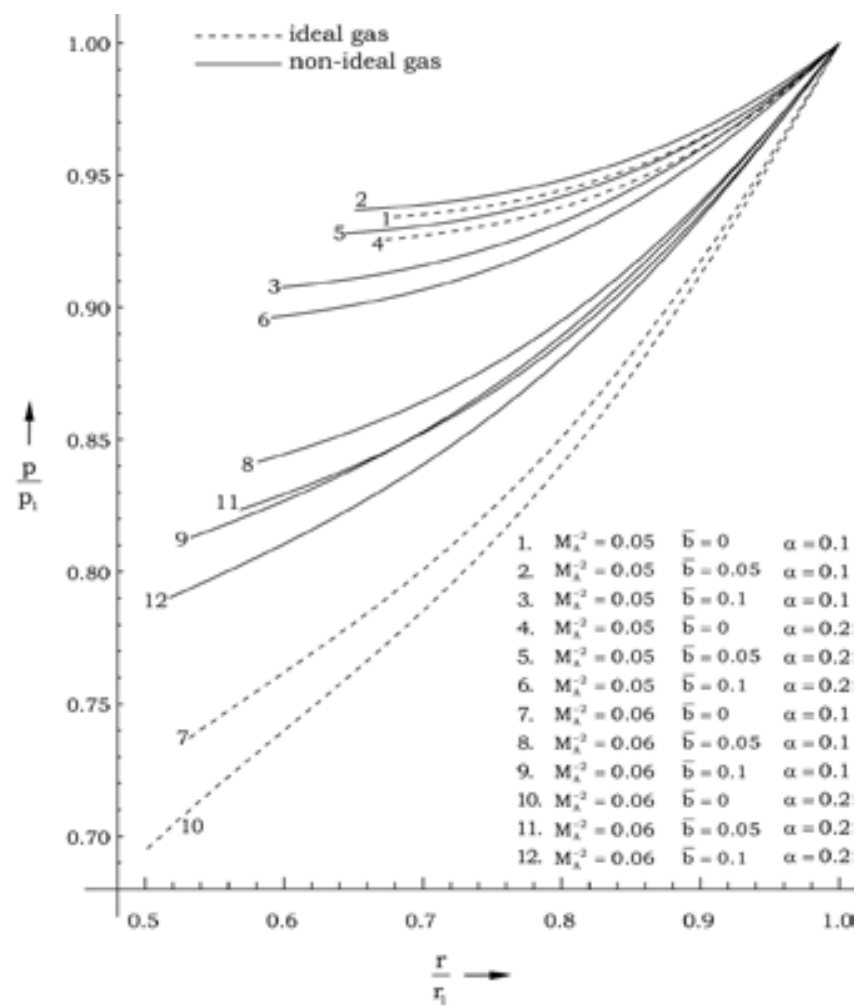

Figure 4. Variation of axial magnetic field $\mathrm{p} / \mathrm{p}_{1}$ with radial distance $\mathrm{r} / \mathrm{r}_{1}$ in the flow-field behind the shock for different values of Alfven-Mach number $M_{A}^{-2}$, parameter of non-idealness of the gas $\bar{b}$ and parameter of radiation

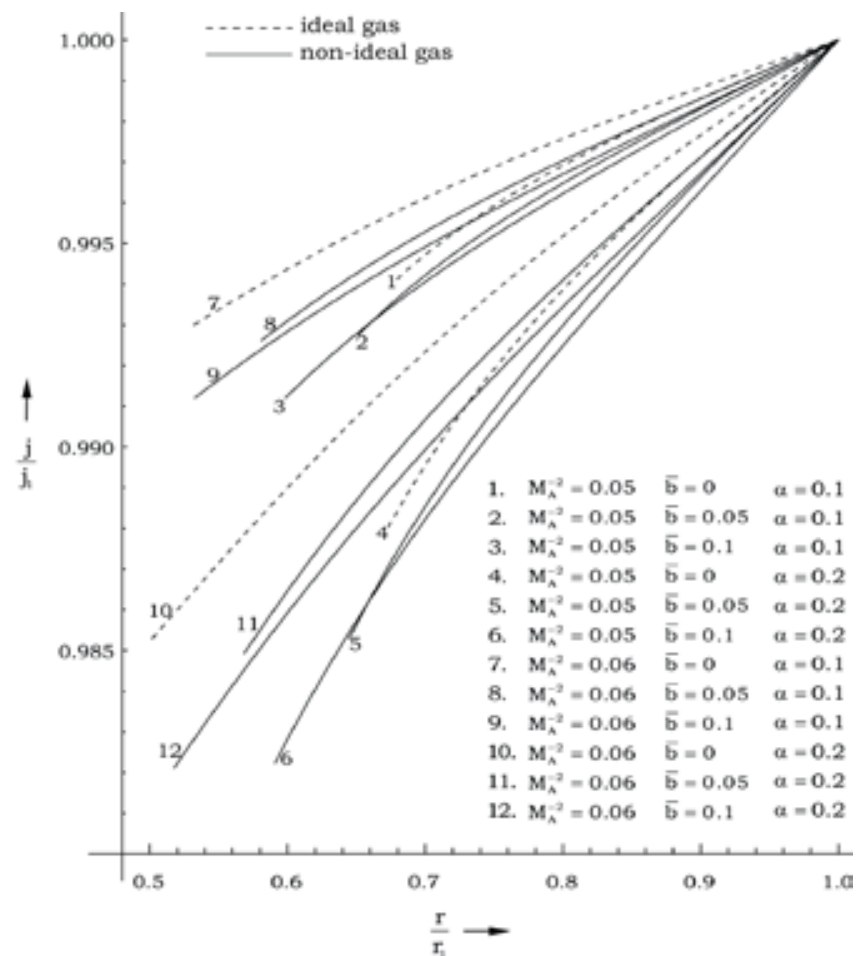

Figure 5. Variation of axial magnetic field $\mathrm{j} / \mathrm{j}_{1}$ with radial distance $\mathrm{r} / \mathrm{r}_{1}$ in the flow-field behind the shock for different values of Alfven-Mach number $M_{A}^{-2}$, parameter of non-idealness of the gas $\bar{b}$ and parameter of radiation $\alpha$

Table 1. Values of density ratio across the shock for different values of $\bar{b}$ and $\mathbf{M}_{\mathrm{A}}^{-2}$

\begin{tabular}{|c|c|c|}
\hline \multirow{2}{*}{$\overline{\mathrm{b}}$} & \multicolumn{2}{|c|}{$\frac{\rho_{0}}{\rho_{1}}=\beta$} \\
\cline { 2 - 3 } & $\mathrm{M}_{\mathrm{A}}^{-2}=0.05$ & $\mathrm{M}_{\mathrm{A}}^{-2}=0.06$ \\
\hline 0 & 0.272955 & 0.278674 \\
\hline 0.05 & 0.305236 & 0.309878 \\
\hline 0.1 & 0.340960 & 0.345155 \\
\hline
\end{tabular}

Table 2. Position of inner expanding surface $x_{p}$ for $\gamma=5 / 3$ and various values of $M_{A}^{-2}, \bar{b}$ and $\alpha$

\begin{tabular}{|c|c|c|c|}
\hline \multirow{3}{*}{$\mathbf{M}_{\mathrm{A}}^{-2}$} & $\alpha$ & $\overline{\mathrm{b}}$ & $\mathbf{x}_{\mathrm{p}}$ \\
\hline \multirow{3}{*}{0.05} & \multirow{3}{*}{0.1} & 0 & 0.679286 \\
\cline { 3 - 4 } & & 0.05 & 0.650849 \\
\cline { 3 - 4 } & \multirow{3}{*}{0.2} & 0.1 & 0.598177 \\
\hline & & 0 & 0.673043 \\
\hline & \multirow{3}{*}{0.06} & 0.05 & 0.644710 \\
\hline & & 0 & 0.590655 \\
\cline { 3 - 4 } & & 0.05 & 0.531759 \\
\cline { 3 - 4 } & \multirow{3}{*}{0.2} & 0.1 & 0.580765 \\
\cline { 3 - 4 } & & 0.05 & 0.532257 \\
\cline { 3 - 4 } & & 0.1 & 0.568326 \\
\hline
\end{tabular}




\section{Conclusions}

The present work investigates the self-similar flow of a non-ideal gas under the action of monochromatic radiation behind a cylindrical shock wave in presence of a uniform axial magnetic field. The density of the ambient medium is also uniform. On the basis of this work, one may draw the following conclusions:

(i) The presence of magnetic field decays the shock wave and enhances the effect of non-idealness of the gas on the pressure profiles behind the shock.

(ii) The non-idealness of the gas decays the shock wave, and affects the velocity and pressure profiles significantly when the initial magnetic field is strong.

(iii) An increase in $\alpha$ decreases the shock strength and the fluid velocity, the pressure and the radiation flux at any point in the flow-field behind the shock, and these behavior of the flow-variables show that the monochromatic radiation is more absorbed. These effects are significant when the initial magnetic field is strong.

Thus it is inferred that the effect of the non-idealness of the gas and of the monochromatic radiation on the shock propagation become more significant when the strength of the initial magnetic field is increased.

\section{REFERENCES}

[1] Sedov, L. I., 1959, Similarity and Dimensional Methods in Mechanics, Academic Press London.

[2] Marshak, R. E., 1958, Effect of radiation on shock wave behaviour, Phys. Fluids, 1, 24-29.

[3] Elloitt, L. A., 1960, Similarity methods in radiation and hydrodynamics, Proc. R. Soc. Lond. A, 258, 287-301.

[4] Wang, K. C., 1964, The 'piston problem' with thermal radiation, J. Fluid Mech., 20, 447-455.

[5] Helliwell, J. B., 1969, Self-similar piston problem with radiative heat transfer, J. Fluid Mech., 37, 497-512.

[6] Nicastro, J. R., 1970, Similarity analysis of radiative gasdynamics with spherical symmetry, Phys. Fluids, 13, 2000-2006.

[7] Deb Ray, G. and Bhowmick, J. B., 1976, Similarity solutions for expansions in stars, Ind. J. Pure Appl. Math., 7, 96-103.

[8] Khudyakov, V. M., 1983, The self-similar problem of the motion of a gas under the action of monochromatic radiation, Sovit Phys. Dokl. (trans. American Institute of Physics), 28, 853-855.

[9] Zheltukhin, A. N., 1968, A family of exact solutions of the equation of the one dimensional motion of a gas under the influence of monochromatic radiation, J. Appl. Math. Mech. (trans. of PMM) 52, 262-263.

[10] Nath, O. and Takhar, H. S., 1990, Propagation of cylindrical shock waves under the action of monochromatic radiation, Astrophys. Space Sci., 166, 35-39.

[11] Nath, O., 1998, Propagation of cylindrical shock wave in a rotating atmosphere under the action of monochromatic radiation, IL Nuovo Cimento D, 20, 1845-1852.

[12] Pai, S. I., 1958, Cylindrical shock waves produced by instantaneous energy release in magnetogasdynamics, Proc. Theo. Appl. Mech., 81, 89-100.

[13] Cole, J. D. and Greifinger, C., 1962, Similarity solution for cylindrical magnetogasdynamic blast waves. Phys. Fluids, 5, 1597-1607.

[14] Sakurai, A., 1965, Blast wave theory, Basic Developm ents in Fluid Dynamics, Vol. 1 (edited by M. Holt), 309-375.

[15] Bhutani, O. P., 1966, Propagation and attenuation of a cylindrical blast wave in magnetogasdynamics, J. Math. Anal. Appl., 13, 565-576.

[16] Christer, A. H. and Helliwel, J. B., 1969, Cylindrical shock and detonation waves in magnetogasdynamics, J. Fluid Mech., 39, 705-725.

[17] Deb ray, G., 1973, Similarity solution for cylindrical blast waves in magnetogasdynamics, Phys. Fluids, 16, 559-560.

[18] Vishwakarma J. P. and Yadav, A. K., 2003, Self-similar analytical solution for blast wave in inhomogeneous atmosphere with frozen-in-magnetic field, Eur. Phys. J. B, 34, 247-253.

[19] Nath, O., 1989, A study of self-similar cylindrical MHD shock waves in monochromatic radiation, Astrophys. Space sci. 155, 163-167.

[20] Shinde, S., 2006, Propagation of cylindrical shock waves in a non-uniform rotating stellar atmosphere under the action of monochromatic radiation and gravitation, Mathe. Comput. Applic., 11, 95-102.

[21] Roberts, P. H. and Wu, C. C., 1996, Structure and stability of a spherical implosion, Phys., Lett. A, 213, 59-64.

[22] Wu, C. C. and Roberts, P. H., 2003, The shock wave theory of sonoluminescence, Shock Focussing Effect in Medical Science and Sonoluminescence (Eds. R. C. Srivastava et al.), Springer-Verlag, Berlin, 1-27.

[23] Zedan, Hassan A., 2002, Application of the group of equations of the one-dimensional motion of a gas under the influence of monochromatic radiation, Appl. Math. Comput., 132, 63-71.

[24] Ranga Rao M. P. and Purohit, N. K., 1976, Self-similar pistion problem in non-ideal gas, Int. J. Engng. Sci., 14, 91-97.

[25] Singh, L. P., Husain, A. and Singh, M., 2011, A selfsimilar solution of exponential shock waves in non-ideal magnetogasdynamics, Meccanica, 46, 437-445.

[26] Vishwakarma, J. P. and Singh, M., 2012, Self-similar cylindrical ionizing shock waves in a non-ideal gas with radiation heat-flux, Applied Mathematics, 2, 1-7. 